\title{
Mineraçãåo
}

\section{Uma heurística para o planejamento operacional de lavra com alocação dinâmica de caminhões}

\section{A heuristic for the open-pit mining operational planning problem with dynamic truck allocation}

Francisco César Rodrigues de Araújo PPGEM/EM/UFOP, Ouro Preto (MG)

e Prof. IFMG - Ouro Preto (MG)

fcesaraujo@yahoo.com.br

Marcone Jamilson Freitas Souza

Prof. Associado, PPGEM/EM/UFOP

e DECOM/ICEB/UFOP Ouro Preto (MG)

marcone@iceb.ufop.br

\section{Resumo}

Esse trabalho trata do planejamento operacional de lavra em minas a céu aberto com alocação dinâmica de caminhões. Esse problema consiste em determinar o número de viagens que cada caminhão deve fazer a cada frente de lavra, bem como decidir em quais frentes de lavra alocar as carregadeiras, de sorte a atender as metas de produção e qualidade requeridas para o minério a ser produzido, fazendo o melhor aproveitamento da frota de veículos disponível. Dada sua complexidade combinatória, o problema é resolvido por meio de um algoritmo heurístico baseado na metaeurística Iterated Local Search. O algoritmo proposto é testado usando-se dados reais e seus resultados são comparados com aqueles encontrados pelo otimizador LINGO aplicado a um modelo de programação matemática.

Palavras-chave: Planejamento operacional de lavra em minas a céu aberto, alocação de caminhões, Iterated Local Search, descida em vizinhança variável, metaeurísticas.

\section{Abstract}

This work addresses open-pit mining operational planning with dynamic truck allocation. The problem involves determining the number of trips that each mining truck would do to each mining face, as well as deciding to which face the shovels are to be allocated, in order to meet the production and quality targets, and to achieve the best utilization of the available fleet. Due to the combinatorial complexity of the problem, it is solved using a heuristic algorithm based on the Iterated Local Search metaheuristic. The proposed algorithm is tested using real data and the results are compared against those produced by a LINGO-based mathematical programming model.

Keywords: Open-pit mine operational planning, truck allocation, Iterated Local Search, variable neighborhood search, metaheuristics.

\section{Introdução}

Devido à competitividade global, que exige das empresas soluções eficientes para seus processos produtivos, as técnicas de pesquisa operacional vêm sendo cada vez mais aplicadas. Na mineração, em particular no planejamento de lavra, a realidade não é diferente. Vários são os sistemas que fazem uso dessas técnicas, como, por exemplo, DISPATCH (www.mmsi. com/DISPATCH.html) e SMART MINE (http://www.devex.net). A questão é que o acesso à descrição pormenorizada das 
técnicas usadas por essas ferramentas não é disponibilizado, por tais técnicas se constituírem em segredo industrial. Entretanto sabe-se que técnicas de programação linear e heurísticas fundamentam tais sistemas.

Estudos como os de White e Olson (1986), Chanda e Dagdelen (1995), Alvarenga (1997), Pinto e Merschmann (2001), Merschmann (2002), Costa et al. (2004, 2005) e Costa (2005) descrevem formulações de programação matemática e/ou algoritmos heurísticos para resolver problemas inerentes ao planejamento operacional de lavra. Em White e Olson (1986), base do sistema DISPACH, a programação de lavra é feita resolvendose dois problemas de programação linear seqüencialmente. No primeiro, procura-se determinar o ritmo de lavra em cada frente, levando-se em consideração o atendimento à qualidade da mistura e às taxas de alimentação da usina de beneficiamento, entre outros itens. No segundo problema, conhecido o ritmo em cada frente, procurase determinar o fluxo ótimo de transporte na mina. Nesse modelo, os autores não levam em consideração metas de produção e qualidade e, somente após definido o ritmo de lavra ideal, em cada frente (resultado do primeiro problema), é que levam em consideração as características dos equipamentos de carga e transporte disponíveis. Chanda e Dagdelen (1995) propõem um modelo de programação matemática que objetiva atender às metas de qualidade e produção. No entanto, não consideram alocação de equipamentos de carga e transporte. O trabalho de Alvarenga (1997), base do sistema SMART MINE, apoiado em Algoritmos Genéticos, trata apenas do despacho ótimo de caminhões, resumindo-se em sugerir qual o ponto de basculamento ou carregamento ideal em um dado momento e em apresentar a rota para o deslocamento. Pinto e Merschmann (2001) e Merschmann (2002) propuseram dois modelos de programação linear, que se prestam à determinação do ritmo de lavra a ser implementado em cada frente de lavra, levando-se em consideração a qualidade do minério em cada frente, a relação estéril/minério desejada, a produção requerida, as características dos equipamentos de carga e transporte, bem como as características operacionais da mina. Os autores não levam em consideração metas de produção e qualidade. Costa et al. $(2004,2005)$ aperfeiçoaram os modelos anteriores, reduzindo o número de variáveis envolvidas na modelagem, e introduziram na função objetivo o atendimento às metas de produção e qualidade. Tais modelos encontram-se detalhados em Costa (2005), sendo que, nesse último trabalho também é apresentado um algoritmo heurístico, baseado na metaeurística Variable Neigborhood Search (Hansen et al., 2008), para encontrar soluções subótimas dos problemas com melhor qualidade que aquelas produzidas pelos modelos matemáticos em um dado tempo computacional de tomada de decisão.

O presente trabalho apresenta um modelo de programação linear e outro heurístico e representa uma evolução em relação aos modelos de Costa (2005). Inclui-se, na função objetivo, a minimização do número de caminhões necessários ao processo e, nas restrições, considera-se o fato de que os caminhões trabalham limitados a uma taxa de utilização. Além disso, o modelo heurístico proposto considera um maior número de movimentos, permitindo explorar melhor o espaço de soluções do problema.

A utilização de técnicas heurísticas se justifica face à natureza combinatória do problema. Esse fato torna proibitiva sua resolução por técnicas exatas, principalmente quando o tempo para a tomada de decisão é baixo. Apesar de essas técnicas não garantirem a otimalidade, elas conseguem, em geral, produzir soluções de boa qualidade, são de fácil implementação e baixo custo de desenvolvimento. Entre as heurísticas, destacam-se as chamadas metaeurísticas, as quais, ao contrário das heurísticas convencionais, têm caráter geral e são providas de mecanismos para tentar escapar de ótimos locais, ainda distantes de um ótimo global (Glover \& Kochenberger, 2003). O algoritmo heurístico proposto, o qual é baseado na metaeurística Iterated Local Search (Stützle, 2006), é validado pelo modelo de programação matemática.

\section{Descrição do problema de planejamento operacional de lavra}

Para atender às necessidades do mercado, as empresas de mineração necessitam fazer um planejamento estratégico de retirada (explotação) de minério. Para executar essa tarefa, conhecida como Planejamento de Lavra, é necessário se conhecer com riqueza de detalhes a área a ser explorada e explotada.

Normalmente, um planejamento é elaborado considerando-se três fases: longo, médio e curto prazo. Na primeira fase, a de longo prazo, faz-se um planejamento mais amplo. Por exemplo, pensa-se na mina para alguns anos. Na segunda, a de médio prazo, faz-se um planejamento para até um ano e, na terceira fase, a de curto prazo, trabalha-se num planejamento diário, mensal e até trimestral. É importante salientar que esses intervalos de tempo não são rígidos e, portanto, podem variar de uma empresa para outra.

O planejamento de curto prazo é, normalmente, realizado por profissionais de Geologia, Engenharia de Minas, Qualidade, Produção e Administração. Essa equipe se interage com o objetivo final de atender ao cliente e é a mais exigida, tendo em vista a necessidade de se cumprir tudo aquilo que fora preestabelecido pelas equipes responsáveis pelo longo e médio prazo.

De posse dos dados relativos à quantidade e à qualidade do minério disponível na mina, pode-se falar em vida útil desta. Essa é uma tarefa das equipes de médio e longo prazo, que objetivam fazer o planejamento para atender ao mercado por um dado período. Evidentemente que, em função do avanço da tecnologia, essa vida útil pode variar. Material antes considerado estéril poderá, no futuro, ser economicamente aproveitado como minério. Em contrapartida, equipamentos de maior produtividade e softwares mais eficazes poderão fazer com que a mina seja exaurida mais rapidamente.

De posse dessas informações, a equi- pe de curto prazo planeja como atender às necessidades por um dado período (até três meses, no caso da empresa analisada). O planejamento é feito para o dia, o mês e o trimestre.

Com a ajuda de softwares elaborados para esse fim, a equipe do planejamento determina aos diversos setores envolvidos (Qualidade, Sistema de Despacho e Produção) quais são as frentes a serem lavradas bem como quais e quantos equipamentos serão envolvidos (operação de alocação de máquinas e caminhões). $\mathrm{O}$ objetivo é elaborar um plano diário de tal forma que, ao final do mês e, também, do trimestre, as metas sejam atingidas tanto em qualidade quanto em quantidade.

Esse último problema, conhecido como planejamento operacional de lavra ou, simplesmente, programação de lavra, envolve, pois, a seleção de frentes a serem lavradas, com seus respectivos ritmos de lavra, a alocação de equipamentos de car- 
ga para elas, bem como o número de viagens que cada caminhão deve fazer a cada frente, de forma a gerar uma quantidade de produto com determinadas especificações. Para a retirada de material (minério ou estéril) das frentes, considera-se uma frota limitada de equipamentos de carga, sendo que estes devem ser alocados às frentes de lavra e operarem em uma faixa de produtividade que torne economicamente viável sua utilização. Considera-se, ainda, que o transporte do material retirado de cada frente de lavra é realizado por uma frota de caminhões com capacidades de carga diferentes. Supõe-se o sistema de alocação dinâmica, isto é, a cada descarga de material, o caminhão pode ser direcionado a uma frente diferente onde haja um equipamento de carga compatível. Essa estratégia faz aumentar a produtividade da frota e proporciona, segundo Costa (2005), um aumento na capacidade de produção da mina ou a redução do número de equipamentos necessários para manter o mesmo nível de produção. $\mathrm{O}$ ritmo de lavra é determinado para uma hora de produção, sendo replicado até uma frente exaurir ou ocorrer uma parada de equipamento, seja por quebra ou manutenção, situação na qual deve ser feita outra programação. No problema abordado, o objetivo é gerar um produto que atenda as metas de produção e qualidade e que necessite de um número reduzido de caminhões.

$\mathrm{Na}$ determinação da programação de lavra, também devem ser levadas em consideração questões como: a variabilidade nos tempos de ciclo dos caminhões, a possibilidade de ocorrência de filas nas frentes e quebras de equipamentos, a prioridade na seqüência de lavra, entre outras. Para alcançar esse objetivo, podese proceder como em Guimarães et al.
(2007). Esses autores propõem um modelo de simulação computacional para validar a programação de lavra gerada por um modelo de otimização. Caso essa programação resultante da otimização não possa ser cumprida, é feita nova programação, tendo-se em vista uma meta de produção menor. O procedimento de otimizar e simular é interrompido somente quando os resultados da otimização forem validados pela simulação.

É importante destacar que, para a alocação dos caminhões, durante a operação, é necessário um sistema computadorizado de despacho, que não é objeto da presente pesquisa. Tal sistema tem por objetivo cumprir a programação estabelecida, levando em consideração a variabilidade dos tempos de ciclo dos caminhões, a ocorrência de filas nas frentes, a disponibilidade de caminhões e carregadeiras em operação, entre outros fatores.

\section{Modelagem heurística do problema abordado}

\section{Iterated local search}

Iterated Local Search (ILS) é uma metaeurística de busca local e, como tal, é baseada na noção de vizinhança. A exploração do espaço de soluções é feita por meio de movimentos, os quais consistem em modificações feitas na solução corrente, guiando para soluções vizinhas. O ILS é baseado na idéia de que um procedimento de busca local pode ser melhorado a partir de perturbações na solução ótima local. A perturbação precisa ser suficientemente forte para permitir que a busca local explore diferentes soluções, mas também fraca o suficiente para evitar um reinício aleatório (Stützle, 2006).

Para aplicar um algoritmo ILS, quatro componentes têm de ser especificadas:

(a) Procedimento GeraSoluçãoInicial(), que gera uma solução inicial s' para o problema.

\section{Busca local}

A Busca Local do ILS é feita pelo método Variable Neighborhood Descent - VND (Hansen \& Mladenovic, 2001; Hansen et al., 2008). O VND é um método de busca local que consiste em explorar o espaço de soluções através (b) Procedimento BuscaLocal(), que retorna uma solução melhorada s".

(c) Procedimento Perturbação(), que modifica a solução corrente $s$ guiando para uma solução intermediária s'.

(d) Procedimento CritérioAceitação(), que decide de qual solução a próxima perturbação será aplicada.

$\mathrm{Na}$ adaptação proposta para o ILS, o algoritmo parte de uma solução inicial $s_{0}$ gerada conforme descrito na subseção 3.6. A seguir, é feita uma busca local, de acordo com a subseção 3.2 , gerando uma solução refinada $s$. A partir de $s$, é obtida uma solução intermediária s' pela aplicação de uma perturbação tal como especificado na subseção 3.8, seguida de novo refinamento. A nova solução $s$ ", fruto do refinamento de s', é aceita como nova solução corrente $s$, caso haja melhora no valor da função de avaliação (descrita na subseção 3.7). Em caso de não haver melhora, volta-se à solução corrente $s$ e aplica-se o mesmo tipo de perturbação, seguida de novo refinamento, por um número máximo de vezes, dado por $10 \%$ do produto do número de frentes pelo número de caminhões. Esgotada essa quantidade de aplicações da perturbação em uso sem que haja melhora global, passa-se para outro tipo de perturbação. Sempre que o algoritmo encontra uma solução refinada de menor custo global, volta-se ao primeiro tipo de perturbação. As perturbações são dispostas de forma que as primeiras são as de menor complexidade computacional. $\mathrm{O}$ algoritmo é interrompido quando um tempo-limite de processamento é alcançado ou quando o número de iterações sem melhora atingir um valor limiar.

\section{Movimentos e estrutura de vizinhança}

O espaço de soluções de um problema pode ser pesquisado navegando-se de uma solução para outra que esteja em sua vizinhança. Uma vizinhança $N(s)$ de uma solução sé um conjunto de soluções $s$ ' que diferem de $s$ por um movimento 
$m$. Este, por sua vez, é uma modificação feita em uma solução $s$. Nesse trabalho, o espaço de soluções do problema abordado é explorado tendo por base os seguintes movimentos, aplicáveis tanto para as frentes de minério quanto para as de estéril:

\section{Dados de entrada}

Sejam:

$F$ - conjunto de frentes.

$V$ - conjunto de veículos.

$C$ - conjunto de equipamentos de carga.

$T$ - conjunto dos parâmetros de controle (teores de $\mathrm{Fe}, \mathrm{Al}_{2} \mathrm{O}_{3}$ e etc e granulometria) presentes nas frentes.

\section{Representação de uma solução}

Uma solução é representada pela matriz $\mathrm{R}_{|\mathrm{F}| \times(1+\mathrm{V})}=\left[\mathrm{Y}_{|\mathrm{F}| \times 1} \mid \mathrm{N}_{|\mathrm{F}| \times|\mathrm{V}|}\right]$, sendo que yik $\in \mathrm{Y}_{|\mathrm{F}| \times 1}$ representa a alocação do equipamento de carga $k$ à frente $\underline{\mathrm{i}}$ (assumindo valor 1 , se o equipamento

\section{Geração de uma solução inicial}

Para se gerar a solução inicial, é necessária a construção das matrizes $\mathrm{Y}_{|\mathrm{F}| \times 1}$, $\mathrm{N}_{|\mathrm{F}| \times|\mathrm{V}|}, \mathrm{S}_{|\mathrm{F}| \times 1}$ e $\mathrm{TcV}_{1 \times|\mathrm{V}|}$. Para se gerar $\mathrm{Y}|\mathrm{Fl}|$, inicialmente, atribui-se o valor -1 para todas as carregadeiras, ou seja, considera-se que não existe carregadeira alocada a nenhuma frente. Atribui-se, também, o valor 0 (zero) para o status de cada carregadeira, significando a inatividade de cada uma delas em cada frente. Em seguida, as carregadeiras são alocadas aleatoriamente a cada uma das frentes disponíveis. Para essas carregadeiras, atribui-se o status ativo (valor 1), inicialmente.

O número de viagens em cada célula da matriz $\mathrm{N}_{|\mathrm{F}| \times|\mathrm{V}|}$ é gerado aleatoriamente. Para cada célula $n_{i l}$ é calculado, inicialmente, o número máximo de viagens (nv_max), por hora, em função do tempo de ciclo (Tc) de cada caminhão $l$ às diversas frentes $i$. É gerado, então, de forma aleatória, um número compreendido entre 0 e nv_max.

Posteriormente, verifica-se se existe alguma carregadeira $k$ alocada a essa frente e se há compatibilidade entre ela e o caminhão $l$ designado. Caso afirmativo, verifica-se se essa carregadeira encontrase ativa ou não. Em caso de estar ativa, toma-se o número aleatório calculado anteriormente como sendo o número de viagens do caminhão $l$ à frente $i$. Caso não esteja ativa ou não havendo compatibilida-
(1) Retirar uma viagem de um caminhão em uma frente qualquer.

(2) Retirar duas viagens de um caminhão em uma frente qualquer.

(3) Realocar uma viagem a uma frente diferente, mantendo o caminhão.

(4) Realocar uma viagem para um

$Q u_{i}$ - quantidade máxima de massa lavrável em cada frente $i$.

$t c_{i l}$ - tempo de ciclo de cada veículo $l$ a cada frente $i$.

$t_{i j}$ - percentual do parâmetro de controle $j$ presente na frente $i$.

Compatib $_{|\mathrm{Cl}| \mathrm{V} \mid \mathrm{V}}$ - matriz que representa a compatibilidade entre os equipamentos

está ativo, e 0 , caso contrário) e $n_{i l} \in$ $\mathrm{N}_{|\mathrm{F}| \times|\mathrm{V}|}$ indica o número de viagens do caminhão $l$ à frente $i$.

A partir de $\mathrm{Y}, \mathrm{N}$ e dos tempos de ciclo da matriz $\mathrm{Tc}_{|\mathrm{F}| x|\mathrm{~V}|}$, são determina-

de entre a carregadeira e o caminhão ou, ainda, não havendo carregadeira alocada à frente, não é atribuída a esse caminhão nenhuma viagem a essa frente. $O$ procedimento é repetido para todas as células da matriz $\mathrm{N}_{|\mathrm{F}| \times|\mathrm{V}|^{\circ}}$.

A quantidade de massa lavrável $\left(s_{i}\right)$ em cada frente $i$ é calculada somando-se o produto do número de viagens pela capacidade $c a p_{l}$ de cada caminhão $l$ que faz viagens à frente.

Para cada caminhão, é calculado o somatório do produto entre o número de viagens $\left(n_{i l}\right)$ e o tempo de ciclo $\left(t c_{i l}\right)$ nas diversas frentes, gerando a matriz $\mathrm{Tcv}_{1 \times \mathrm{V} \mid}$ (somatório dos tempos de ciclo por caminhão). Aplicase, a seguir, um procedimento para verificar a existência de dois tipos de inviabilidade. A correção das inviabilidades propicia uma solução inicial de melhor qualidade, diminuindo o esforço computacional exigido no refinamento dessa solução.

A primeira inviabilidade diz respeito ao somatório dos tempos de ciclo por caminhão. Esse tempo acumulado não poderá exceder a 60 minutos, já que o objetivo é determinar o ritmo de lavra para uma hora. Para cumprir esse objetivo, verifica-se, para cada caminhão $l$, se o tempo de ciclo acumulado $\left(t c v_{l}\right)$ é superior a 60 minutos. Caso seja, é determinada uma frente $i$, aleatoriamente, e retirada uma viagem caminhão diferente, mantendo a frente.

(5) Inserir uma viagem de um caminhão a uma frente.

(6) Retirar todas as viagens de um caminhão em todas as frentes.

(7) Desativar uma carregadeira.

(8) Realocar uma carregadeira.

de carga $k$ e os caminhões $l$.

cap $p_{l}$ - capacidade de cada caminhão $l$.

$\mathrm{Cl}_{k}$ - capacidade mínima de cada carregadeira $k$.

Cuk - capacidade máxima de cada carregadeira $k$.

TxMax - taxa de utilização máxima permitida para o $l$-ésimo caminhão.

$\operatorname{dos} \mathrm{S}_{|\mathrm{F}| \times \mid}$ e $\mathrm{Tcv}_{1 \times|\mathrm{V}|}$, os quais representam, respectivamente, a quantidade de massa lavrada em cada frente e o somatório dos tempos de ciclo de cada veículo.

do caminhão $l$, caso exista. Em seguida, subtrai-se o tempo de ciclo $\left(t c_{i, l}\right)$ correspondente. $O$ procedimento é repetido, para cada caminhão, até que o tempo acumulado seja inferior ou igual a 60 minutos. Após aplicar esse reparador de inviabilidade, é necessário reconstruir a matriz $\mathrm{S}_{|\mathrm{F}| \times \mid}$, que contém a massa lavrável $s_{i}$ em cada frente $i$.

A segunda inviabilidade diz respeito à quantidade de massa lavrável, por frente. Se essa quantidade for superior à massa $Q u_{i}$ disponível na frente $i$ ou superior à capacidade máxima horária de produção da carregadeira alocada à frente $i$, então é escolhido aleatoriamente um caminhão $l$. Caso haja viagens desse caminhão $l$ à frente $i$, é retirada uma viagem dele e recalculados $t c v_{l}$ e $s_{i}$. É atualizada também a variável que corresponde à somatória de todas as massas lavráveis, seja de minério, seja de estéril. Esse procedimento é repetido para todas as frentes até eliminar a ocorrência deste tipo de inviabilidade.

Esse processo de geração de uma solução inicial é repetido IterConstr vezes e, em cada uma delas, aplicado o método VND descrito na subseção 3.2. A melhor solução obtida é usada como solução inicial para o ILS. Esse procedimento tem a vantagem de produzir uma solução inicial de boa qualidade rapidamente, facilitando a fase de refinamento. 


\section{Avaliação de uma solução}

Uma solução $s$ é avaliada em função da produção, da qualidade do produto

em que $f^{p}(s)$ avalia $s$ quanto à produção; $f_{i}^{q}(s)$ faz a avaliação quanto à qualidade do $j$-ésimo parâmetro de controle; $f_{l}^{u}(s)$ avalia $\mathrm{o}$ atendimento da taxa de utilização máxima do $l$-ésimo caminhão; $f^{l}(s)$ é a componente que avalia $s$ quanto ao núme-

\section{- Produção de minério estéril}

A produção de minério e estéril é

avaliada pela Eq. (2):

$$
f^{p}(s)=\alpha \times(|\operatorname{Pr}-P|+\gamma \times|E r-P e|)
$$

em que $P$ é a produção de minério (t), $P e$, a de estéril (t), $P r$ é a produção recomendada de minério $(\mathrm{t}), E r$, a produção recomendada de estéril (t) e $\alpha$ e $\gamma$

são penalidades por desvios de produção de minério e estéril, respectivamente. A produção $P$ é calculada pela Eq. (3). Para o cálculo de $P e$, substitui-se o

$$
P=\sum_{i \in M} \sum_{l \in V} n_{i l} \operatorname{cap}_{l}
$$

\section{- Qualidade da mistura}

A qualidade da mistura é avaliada segundo a Eq. (4), em que $t m_{j}$ é o valor percentual encontrado para o parâme-

O valor $t m_{j}$ é calculado através da média ponderada entre $t_{i j}$ e a produção

tro $j(\%)$ na mistura; $t r_{j}$ é a meta de qualidade para o parâmetro $j(\%) ; P$ indica a produção de minério $(\mathrm{t})$ e $\beta_{j}^{q}$

$$
f_{j}^{q}(s)=\beta_{j}^{q} \times P \times\left|t r_{j}-t m_{j}\right| / 100 \quad \forall j \in T
$$

(em uma hora) de cada frente $i$ de minério, conforme mostrado na Eq. (5).

$$
t m_{j}=\sum_{i \in M} t_{i j} x_{i} / \sum_{i \in M} x_{i} \quad \forall j \in T
$$

O ritmo de lavra $x_{i}$ em cada frente $i$

é calculado com base na Eq. (6).

$$
x_{i}=\sum_{l \in V} n_{i l} \operatorname{cap}_{l}
$$

\section{- Taxa de utilização de caminhões}

A utilização dos caminhões é avaliada pela equação (7), em que TxUtil $l_{l}$ representa a taxa de utilização

do caminhão $l$, calculada pela Eq. (8),

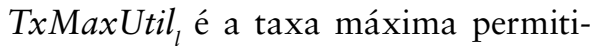
penalidade por se ultrapassar essa taxa máxima. é a penalidade por não atendimento à meta de qualidade do parâmetro de controle $j$;

conjunto das frentes de minério $M$ pelo conjunto $E$ das frentes de estéril. da de utilização do caminhão $l$ e $\mu$ é a

$$
\begin{array}{ll}
f_{l}^{u}(s)=\mu \times \mid \text { TxUtil }_{l}-\text { TxMaxUtil }_{l} \mid & \forall l \in V \\
\text { TxUtil }_{l}=\sum_{i \in F} n_{i l} \text { tc }_{i l} \quad \forall l \in V &
\end{array}
$$

para o caso de a quantidade transportada estar acima da máxima permitida e um valor baixo $\left(\mu_{B}\right)$, caso contrário. 
- Número de caminhões utilizados

O número de caminhões usados é avaliado pela Eq. (9), em que $u s o u_{l}$ assume valor 1 se o caminhão $l$ é usado e 0 , caso contrário, e $\omega_{l}$ é a penalidade pelo uso do

$$
f^{l}(s)=\omega_{l} \times \sum_{l \in V} \text { ssou }_{l}
$$

caminhão.

\section{- Avaliação da produtividade das carregadeiras}

A produtividade das carregadeiras é avaliada pela Eq. (10), onde $\varphi$ é a pena- lidade por não se atingir a produtividade máxima da carregadeira $k \mathrm{e} \lambda$, a penalidade por utilizar uma carregadeira em uma produtividade inferior à mínima recomendada.

$f_{k}^{c}(s)=\left\{\begin{array}{ll}\varphi \times\left(C u_{k}-\operatorname{prod}_{k}\right) & \text { se } C l_{k} \leq \operatorname{prod}_{k} \leq C u_{k} \\ \lambda \times\left(C l_{k}-\operatorname{prod}_{k}\right) & \text { se } \operatorname{prod}_{k}<C l_{k}\end{array} \quad \forall k \in C\right.$

\section{Perturbações}

Para tentar escapar de ótimos locais ainda distantes do ótimo global, o método Iterated Local Search faz uso de perturbações. Uma perturbação nada mais é do que uma modificação feita em uma solução para funcionar como um mecanismo de diversificação do algoritmo. As seguintes perturbações são usadas:

(1) Retirar uma viagem.

\section{Resultados computacionais}

O algoritmo heurístico ILS proposto foi implementado na linguagem visual Delphi, versão 7. Para testar sua eficiência, o mesmo foi comparado com o otimizador LINGO, versão 10, aplicado a um modelo de programação matemática, que representa uma extensão do de Costa et al. (2004). Esse modelo, disponível em http://www. iceb.ufop.br/decom/prof/marcone/projects/ polad.html, tem a equação referente à relação estéril/minério substituída por uma equação de meta de produção de estéril de forma a possibilitar o estabelecimento de limites inferiores e superiores de produ-
(2) Retirar duas viagens.

(3) Realocar viagem mantendo o caminhão.

(4) Realocar viagem mantendo a frente.

(5) Retirar viagem, duas vezes.

(6) Retirar duas viagens, duas vezes.

(7) Realocar viagem mantendo o caminhão, duas vezes.
(8) Realocar viagem mantendo a frente, duas vezes.

(9) Realocar carregadeira.

(10) Desativar carregadeira.

(11) Ativar carregadeira.

(12) Trocar carregadeira.

(13) Tirar todas as viagens de um caminhão.

(14) Inserir uma viagem. ção de estéril diferentes dos de minério. Adicionalmente são incluídas restrições relativas às taxas de utilização dos veículos de transporte, bem como uma componente de avaliação do número de veículos usados. Os dois algoritmos, heurístico e exato, foram testados em um microcomputador com processador AMD Turion64 Mobile, Technology MK-36, 2,01 GHz, com 1 GB de RAM.

Apresentam-se, a seguir, os resultados do uso dessas metodologias em um problema-teste envolvendo 5 parâmetros de controle (\% de Fe, $\left.\mathrm{Al}_{2} \mathrm{O}_{3}, \mathrm{P}, \mathrm{PPC}, \mathrm{He}\right), 15$

\begin{tabular}{c|c|c|c|c|c|c}
\hline Item & Prior. & Meta & ILS & LINGO(1) & LINGO(2) & LINGO(3) \\
\hline$\% ~ F e$ & $\mathrm{MI}$ & 47.00000 & 47.9825 & 47.9889 & 47.9889 & 47.9889 \\
\hline$\% \mathrm{Al}_{2} \mathrm{O}_{3}$ & $\mathrm{I}$ & 0.3200 & 0.3175 & 0.3173 & 0.3173 & 0.3173 \\
\hline$\% \mathrm{P}$ & $\mathrm{MC}$ & 0.0400 & 0.0419 & 0.0419 & 0.0419 & 0.0419 \\
\hline$\%$ PPC & $\mathrm{C}$ & 2.35 & 2.9137 & 2.9154 & 2.9154 & 2.9154 \\
\hline$\%$ He & $\mathrm{I}$ & 40.00 & 37.95 & 37.97 & 37.97 & 37.97 \\
\hline fo & & - & $30,583.83$ & $30,812.70$ & $30,736.99$ & $30,735.99$ \\
\hline \#Carregadeiras & & - & 6 & 6 & 6 & 6 \\
\hline \# Caminhões & & - & 20 & 17 & 18 & 17 \\
\hline \# Viagens & & - & 92 & 71 & 71 & 71 \\
\hline \%Tx. util. cam. & & 85 & 76.98 & 73.5 & 69.42 & 73.5 \\
\hline Tempo & & - & 161 seg. & 161 seg. & $1 \mathrm{~h}$. & $20 \mathrm{~h}$. \\
\hline
\end{tabular}

frentes de lavra, sendo 11 de minério e 4 de estéril, com 30 caminhões (sendo $15 \mathrm{com}$ capacidade de 50 t e outros 15 de 80 t) e 8 carregadeiras de diferentes produtividades. Os dados completos desse cenário, bem como os valores dos pesos considerados na função objetivo e dos parâmetros utilizados pelo ILS estão disponíveis no mesmo endereço apontado anteriormente.

Os dados desse cenário foram submetidos a dez execuções do algoritmo ILS proposto, o que se deve à natureza estocástica do algoritmo proposto. De forma a comparar os resultados por ele gerados 
com aqueles obtidos pelo otimizador LINGO aplicado ao modelo de programação matemática, interrompeu-se a execução deste último em três situações:

(1) No mesmo tempo gasto pelo ILS para gerar a melhor solução.

(2) Após uma hora de processamento

(3) Após 20 horas de processamento.

O objetivo de se aumentar o tempo dado ao LINGO é para verificar a capacidade do ILS em produzir melhores soluções mais rapidamente. Os resultados obtidos

\section{Conclusões}

Esse trabalho tratou o problema de planejamento operacional de lavra considerando alocação dinâmica de caminhões. Dada sua dificuldade de resolução na otimalidade, foi desenvolvido um algoritmo heurístico baseado na metaeurística Iterated Local Search (ILS).

$\mathrm{O}$ algoritmo ILS proposto foi testado em um cenário relativo a um problema real de uma mineração de ferro, comparando-se as soluções produzidas por ele com aquelas geradas por um otimizador aplicado a um modelo de programação matemática desenvolvido.

Os resultados obtidos mostraram que as melhores soluções produzidas pelo ILS são de melhor qualidade que aquelas

\section{Agradecimentos}

Os autores agradecem à FAPEMIG, processos CEX APQ-2991-6.01/07 e CEX PPM 00357/09, e ao CNPq,

\section{Referências bibliográficas}

são apresentados na Tabela 1 . Nessa tabela, a coluna Prioridade indica a prioridade indicada para cada parâmetro de controle, cujos valores possíveis são: Muito Crítico (MC), Crítico (C), Muito Importante (MI), Importante (I) e Irrelevante (-).

Como se observa na Tabela 1, em relação à função objetivo (fo), o ILS produz, em 161 segundos, uma solução melhor que o LINGO, tanto em 161 segundos, quanto em uma hora e, também, em 20 horas de processamento. A solução do ILS é a que mais se aproximou das metas de qualidade e, sendo aquela que apresentou a maior taxa de utilização dos caminhões. A exceção ocorre para o percentual de He. No entanto, esse parâmetro de controle tem prioridade baixa. Com relação ao número de veículos e viagens, as soluções LINGO são mais eficientes; no entanto, na função objetivo, a redução do número de caminhões tem prioridade inferior à da melhora em uma tonelada na qualidade dos parâmetros de controle da mistura. produzidas pelo otimizador LINGO, com relação ao valor da função de avaliação. Adicionalmente, verificou-se que as soluções produzidas pelo ILS foram obtidas sem muito esforço computacional, ao contrário daquelas geradas pelo otimizador de programação matemática. Este último demandou tempo proibitivo para gerar soluções próximas àquelas geradas pelo ILS em poucos minutos. Com relação às características das soluções produzidas pelo algoritmo, pode-se verificar que os valores dos parâmetros de controle ficaram bem próximos das metas estabelecidas. Em relação à frota de veículos de transporte e de equipamentos de carga ficou constatado o bom aproveitamento dos mesmos. No caso dos veículos de transporte, houve um melhor aproveitamento deles, o que é indicado pelas taxas de utilização mais próximas à máxima permitida. Esses resultados validam o uso do ILS enquanto ferramenta de apoio à programação de lavra. Destaca-se, finalmente, que o algoritmo ILS proposto é flexível, no sentido de que outras restrições podem ser facilmente incorporadas. Por exemplo, dada uma prioridade para as frentes de lavra, basta criar uma variável que mensure se as frentes priorizadas estão sendo utilizadas. Caso elas não façam parte da programação de lavra, deve-se incluir um termo na função objetivo para penalizar essa não ocorrência. processo 474831/2007-8, pelo apoio ao desenvolvimento desse trabalho. Agradecem, também, aos revisores anônimos pelos valiosos comentários.

ALVARENGA, G. B. Despacho ótimo de caminhões numa mineração de ferro utilizando algoritmo genético com processamento paralelo. Belo Horizonte: Programa de Pós-Graduação em Engenharia Elétrica, UFMG, 1997. 80 p. (Dissertação de Mestrado).

CHANDA, E. K. C., DAGDELEN, K. Optimal blending of mine production using goal programming and interactive graphics systems. International Journal of Surface Mining, Reclamation and Enviroment, v. 9, p. 203-208, 1995.

COSTA, F. P. Aplicações de técnicas de otimização a problemas de planejamento operacional de lavras em mina a céu aberto. Ouro Preto: Programa de Pós-Graduação em Engenharia Mineral, UFOP, 2005. 141 p. (Dissertação de Mestrado).

COSTA, F. P., SOUZA, M. J. F., PINTO, L. R. Um modelo de alocação dinâmica de caminhões. Revista Brasil Mineral, v. 231, p. 26-31, 2004.

COSTA, F. P., SOUZA, M. J. F., PINTO, L. R. Um modelo de programação matemática para alocação estática de caminhões visando ao atendimento de metas de produção e qualidade. REM-Revista da Escola de Minas, v. 58, p. 77-81, 2005.

GLOVER, F., KOCHENBERGER, G. Handbook of Metaheuristics. Kluwer Academic Publishers, 2003.

GUIMARÃES, I. F., PANTUZA, G., SOUZA, M. J. F. Um modelo de simulação computacional para validar resultados da alocação dinâmica de caminhões em minas a céu aberto. In: SIMPÓSIO DE ENGENHARIA DE PRODUÇÃO - SIMPEP, 14. Anais... Bauru, 2007. 11 p.

HANSEN, P., MLADENOVIC, N. Variable Neighborhood Search: Principles and applica- 
tions. European Journal of Operational Research, v. 130, p. 449-467, 2001.

HANSEN, P., MLADENOVIC, N., PÉREZ, J. A. M. Variable neigborhood search. European Journal of Operational Research, v. 191, p. 593-595, 2008.

MERSCHMANN, L. H. C. Desenvolvimento de um sistema de otimização e simulação para cenários de produção em minas a céu aberto. Rio de Janeiro: Programa de Engenharia de Produção, COPPE/UFRJ, 2002. 93 p. (Dissertação de Mestrado).

PINTO, L. R., MERSCHMANN, L. H. C. Planejamento operacional da lavra de mina usando modelos matemáticos. REM - Revista Escola de Minas, v. 54, n. 3, p. 211-214, 2001.

STÜTZLE, T. Iterated Local Search for the quadratic assignment problem. European Journal of Operational Research, v. 174, p. 1519-1539, 2006.

WHITE, J. W., OLSON, J. P. Computer-based dispatching in mines with concurrent operating objetives. Mining Engineering, v. 38, n. 11, p. 1045-1054, 1986.

Artigo recebido em 22 de março de 2009. Aprovado em 05 de agosto de 2010.

Engenharia Civil | Geologia | Metalurgia e Materiais | Mineraçio
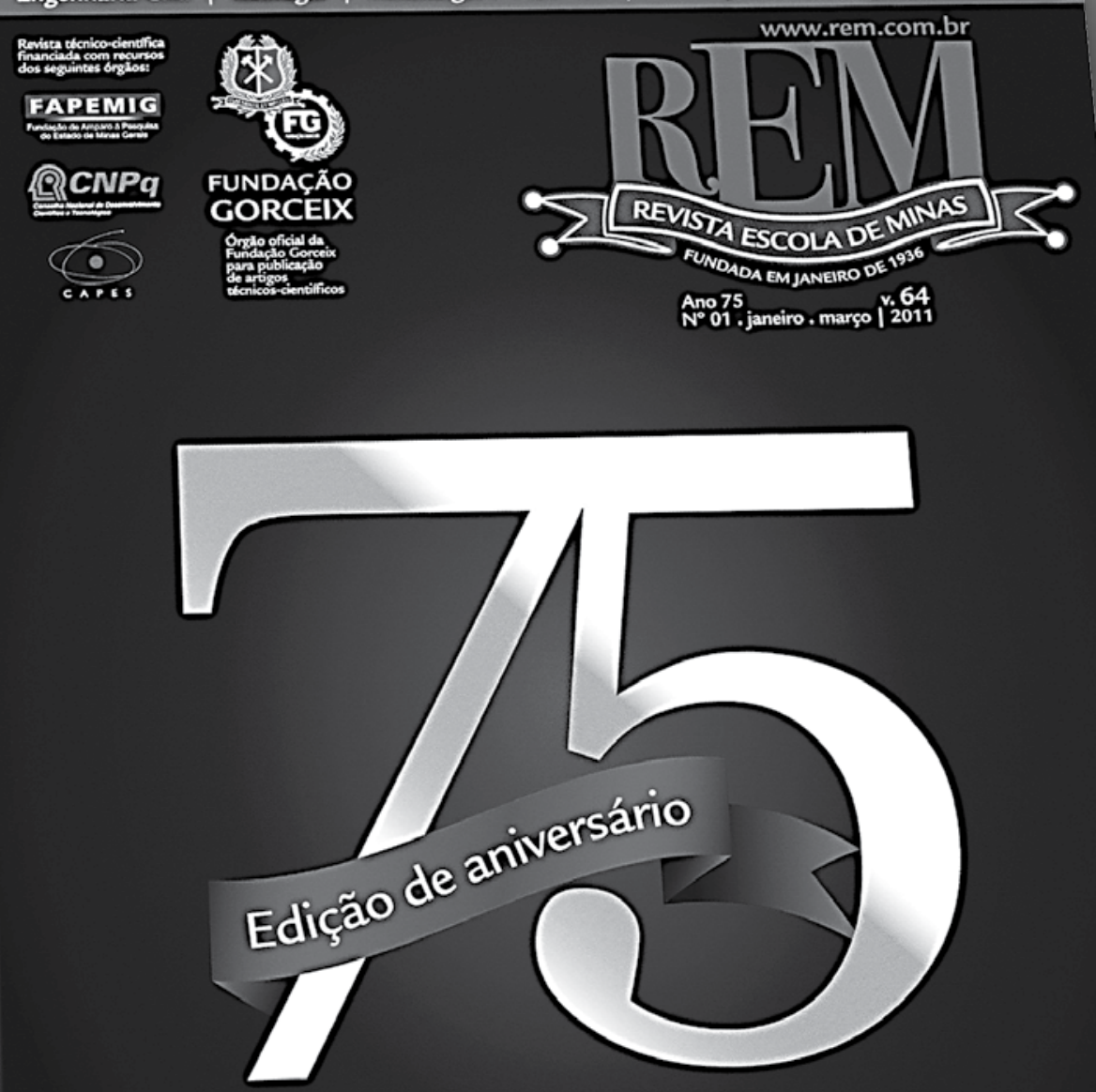

$1936-2011$

\section{ISSN 0370-4467}

\section{(1)}

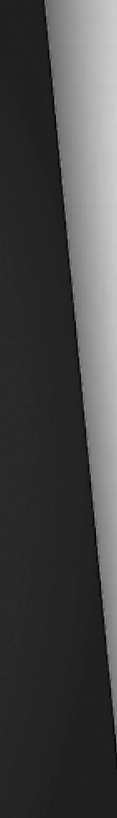

Pessoa física e Bibliotecas - $\mathrm{R} \$ 60,00$

Pessoa Jurídica - R\$80,00

Estudante - R\$30,00

Número Avulso - R\$30,00

Exterior - US\$150,00

A primeira nevista do setor mínero
Economize ao assinar um pacote anual com 4 exemplares trimestrais. mais novos estudos sobre o setor mínero-metalúrgicos do Brasil e do mundo.

Pessoa Juridica - R\$80,00

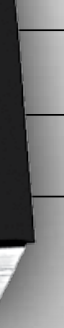

